Pyrenacantha kirkii Baill. (Icacinaceae), nouveau pour le Zaïre

Author(s): F. Malaisse

Source: Bulletin du Jardin botanique National de Belgique / Bulletin van de Nationale

Plantentuin van België, Vol. 57, No. 3/4 (Dec. 31, 1987), pp. 363-366

Published by: Botanic Garden Meise

Stable URL: https://www.jstor.org/stable/3668109

Accessed: 08-01-2022 08:09 UTC

JSTOR is a not-for-profit service that helps scholars, researchers, and students discover, use, and build upon a wide range of content in a trusted digital archive. We use information technology and tools to increase productivity and facilitate new forms of scholarship. For more information about JSTOR, please contact support@jstor.org.

Your use of the JSTOR archive indicates your acceptance of the Terms \& Conditions of Use, available at https://about.jstor.org/terms

Botanic Garden Meise is collaborating with JSTOR to digitize, preserve and extend access to Bulletin du Jardin botanique National de Belgique / Bulletin van de Nationale Plantentuin van België 


\title{
Pyrenacantha kirkii Baill. (Icacinaceae), nouveau pour le Zaïre
}

\author{
par \\ F. Malaisse (*)
}

\begin{abstract}
Pyrenacantha kirkii Baill., known from Zambia and Mozambique, is recorded for the first time in Shaba (Zaire) and a key to the eight species occuring in Zaire is provided.
\end{abstract}

Sept espèces de Pyrenacantha Wight avaient été signalées par R. Boutique dans la Flore du Congo belge et du Ruanda-Urundi (9: 249-257. 1960). Une huitième espèce vient s'ajouter à ce nombre.

Pyrenacantha kirkii Baill., Adansonia 10 : 272 (1872); Mendes, Fl. Zamb. 2: 351 (1963). - Fig. 1.

Liane ligneuse, \pm volubile, atteignant $15 \mathrm{~m}$ de long et $1,2 \mathrm{~cm}$ de diam. à la base, parfois plus petite et plus grêle; tiges âgées gris brunâtre à gris verdâtre, glabres, rugueuses, fréquemment torsadées, légèrement canaliculées; jeunes rameaux densement hirsutes; vieux bois présentant en coupe transversale quelques sillons libériens. Feuilles alternes, à pétiole de 2,5-4(8) $\mathrm{mm}$ de long, recourbé, pubérulent à glabrescent; limbe étroitement elliptiqueobovale, largement arrondi à la base et un peu échancré, atténué à subaigu au sommet, fréquemment mucroné, de $3-7 \mathrm{~cm}$ de long et $1-3,5 \mathrm{~cm}$ de large, un peu discolore, vert et luisant en dessus, vert un peu grisâtre en dessous, à bord entier à parfois irrégulièrement sublobulé, penninerve, à nervures secondaires parfois à fréquemment terminées par des dents glanduleuses situées au bord

(*) Jardin botanique national de Belgique, Domaine de Bouchout, B-1860 Meise (Belgique). - Manuscrit déposé le 25 juin 1987. 


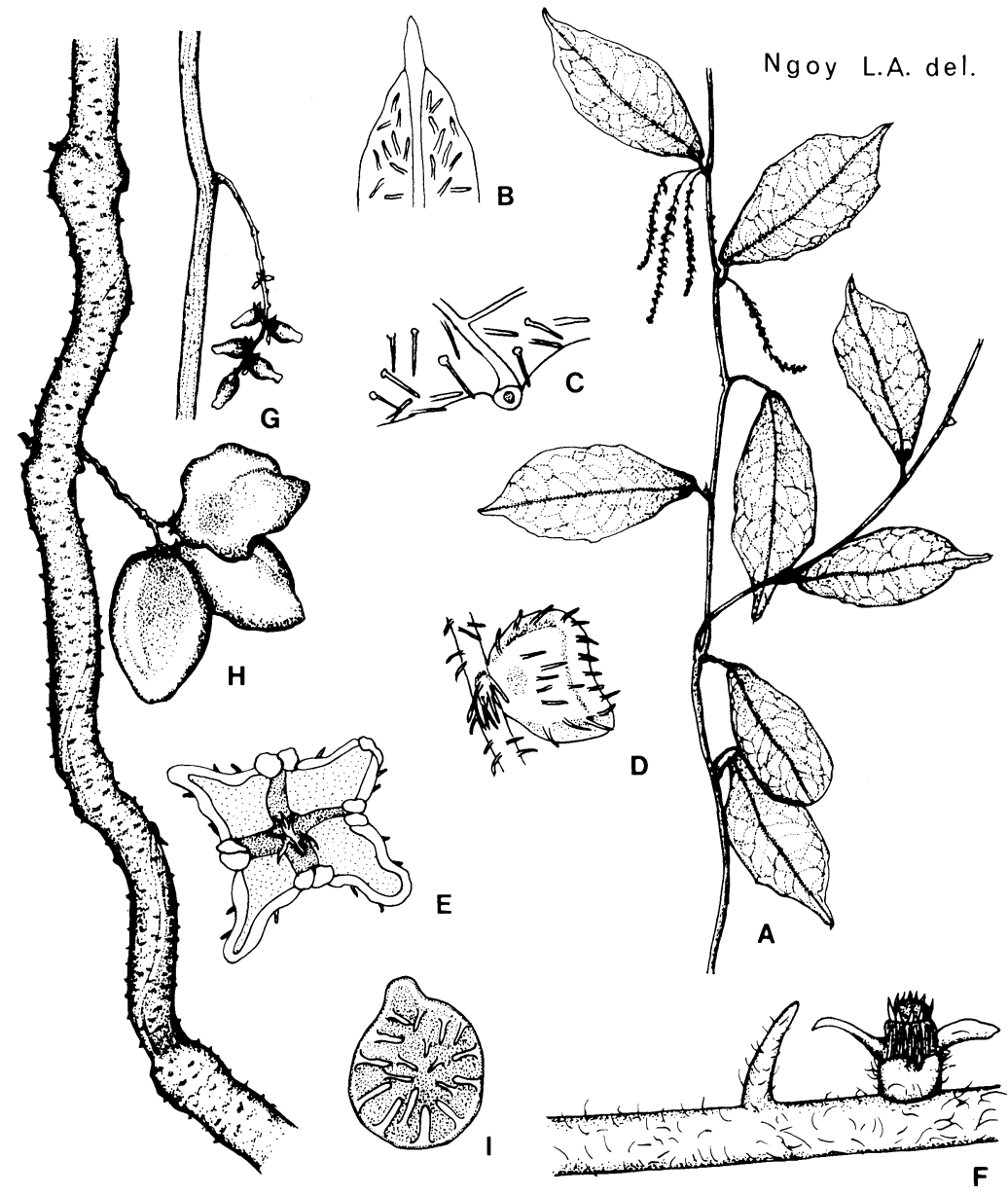

Fig. 1. - Pyrenacantha kirkii Baillon: A, rameau florifère $O^{*}(\times 1 / 2)$; B, sommet du limbe, face abaxiale $(\times 12)$; $C$, bord du limbe avec dent glanduleuse $(\times 20)$; $\mathrm{D}$, bouton floral o* $(\times 15) ; \mathrm{E}$, fleur $\mathrm{O}^{*}(\times 12) ; \mathrm{F}$, fleur ${ }^{\circ}(\times 10)$; G, jeune infrutescence $(X 1) ; \mathrm{H}$, rameau fructifère $(\times 1)$; I, coupe longitudinale montrant la face interne de l'endocarpe $(\times 1,5)$ (A-E, Malaisse 14022; F, Malaisse 11349 bis; G-I, Malaisse 14 023).

du limbe et inclinées vers le bas, à face supérieure glabre, à face inférieure pubescente à poils raides apprimés. Inflorescences spiciformes, solitaires ou groupées en fascicules, axillaires à supraaxillaires, pendantes, grêles, pubescentes, à rachis muni de bractées; inflorescences $\sigma^{\prime}$ de 1,5-4,5 $\mathrm{cm}$ de long; inflorescences o développées sur des rameaux feuillés ou défeuillés, ou caulinai- 
res, de 1,0-2,5 cm de long. Fleurs ơ nombreuses, lâches, sessiles à subsessiles, 4-mères, à bouton de section carrée et aplati au sommet; corolle à $4(5)$ pétales soudés dans le tiers inférieur, rhomboédriques, de $1 \mathrm{~mm}$ de long; étamines à filet de $0,5 \mathrm{~mm}$, soudé à la corolle sur les $2 / 3$ inférieurs; pistillode réduit à une touffe de poils; fleurs $\$$ peu nombreuses, sessiles, sous-tendues par une bractéole couverte extérieurement de longs poils raides; corolle à 4 pétales libres, pubescents extérieurement, glabres intérieurement, elliptiques, de $0,8 \mathrm{~mm}$ de long et $0,6 \mathrm{~mm}$ de large, à sommet obtus; ovaire pubescent, couvert de nombreux poils raides et dressés; style nul; stigmate cupuliforme, à lobes digités. Drupes gris verdâtre, nettement aplaties et à apicule bien différencié à l'état jeune, ellipsoïdes-globuleuses, un peu aplaties et virant de l'orange au brun à maturité, à l'état frais de $1,7 \mathrm{~cm}$ de long, $1-1,1 \mathrm{~cm}$ de large et 0,8-0,9 cm d'épaisseur, pubérulentes, à l'état sec de $1-1,2 \mathrm{~cm}$ de long, de $0,9 \mathrm{~cm}$ de large et $0,5 \mathrm{~cm}$ d'épaisseur; endocarpe à face externe brun grisâtre avec quelques alvéoles peu marquées et à face interne beige munie de longs aiguillons. Graines ellipsoïdes, aplaties, de 9-10 $\mathrm{mm}$ de long et 6-7 $\mathrm{mm}$ de large.

HaUt-Katanga: Lubumbashi-Likasi, $\mathrm{km} 50,5 \mathrm{~km}$ au $\mathrm{N}$ de Shilatembo, Bamps \& Malaisse 8044 ( $\left.0^{\circ}, \mathrm{BR}\right)$, Malaisse 14197 ( $\left.0^{\circ}, \mathrm{BR}\right)$; ibid. km 58, Sofumwango, Malaisse 11649 ( $0^{*}, \mathrm{BR}$ ), 11649 bis ( $($, BR); ibid. km 70, riv. Luafi, Malaisse 11273 (o*, BR), 14022 (o*, BR), 14023 (\%, BR); $5 \mathrm{~km}$ au SE de Kyamasumba, zone Kolwezi, riv. Mushinji (= Mushyshya), Malaisse 14154 (ơ, BR).

Mozambique, Zambie.

Habitat: Forêts denses de ravin.

Observations: 1. - C'est la première fois qu'un Pyrenacantha est récolté dans le district phytogéographique du Haut-Katanga au Zaïre, les sept autres espèces étant connues de la Région guinéo-congolaise, principalement des Districts Forestier Central et du Mayombe.

2. - Mendes (op. cit. : 347) signale, dans sa clé des espèces de Pyrenacantha, l'absence de dents glanduleuses chez P. kirkii. Cellesci sont toutefois présentes sur le matériel shabien, ainsi que sur le matériel zambien déposé à BR (Fanshawe 2970).

3. - Les forêts denses de ravin sont très rares sur les moyens plateaux du Shaba méridional. Lootens (Géo-Eco-Trop 7: 125135. 1983) a décrit la genèse du réseau fluviatile raviné du bassin 
de la Lufira supérieure. La profondeur de ces ravins est généralement comprise entre 7 et $10 \mathrm{~m}$, mais peut atteindre localement $15 \mathrm{~m}$ et plus. Leur profil transversal montre des versants raides avec des pentes rectilignes ou légèrement convexes qui ont la plupart du temps des valeurs supérieures à $40^{\circ}$. Leur intérêt phytogéographique est grand et justifie une prospection approfondie.

4. - Pour la Flore d'Afrique centrale, la clé de détermination peut être modifiée comme suit:

1. Feuilles à pétiole de $5-12 \mathrm{~cm}$ de long; limbe palminerve pourvu d'hydathodes à env. $5 \mathrm{~mm}$ du bord du limbe . . . . 1. P. klaineana var. congolana

1. Feuilles à pétiole ne dépassant guère $4,5 \mathrm{~cm}$ de long; limbe penninerve; hydathodes absents ou sur le bord du limbe:

2. Inflorescences $\sigma^{*}$ et $\uparrow$ subsemblables, racémiformes ou spiciformes, généralement grêles et \pm lâches:

3. Face inférieure des feuilles à poils dressés à subdressés :

4. Présence d'un indument rougeâtre à fauve rougeâtre (surtout sur les parties jeunes, pétioles et drupes) . . . . . . . 2. P. sylvestris

4. Absence d'indument rougeâtre. . . . . . . . . 3. P. puberula

3. Face inférieure des feuilles à poils apprimés à subapprimés:

5. Inflorescence racémiformes

6. Drupe à bec long ( $5 \mathrm{~mm})$; bouton floral on subglobuleux. . .

4. P. acuminata

6. Drupe à apicule court $(1-1,5 \mathrm{~mm})$; bouton floral on oblong. . . . . . . . . . . . . . . . . 5. P. lebrunii

5. Inflorescences spiciformes

7. Drupe à bec long (4-6 mm); feuilles grandes de 7-12,5(17) $\mathrm{cm}$ de long et 3-6(7) cm de large . . . . . . . 6. P. vogeliana

7. Drupe à apicule court $(2-3 \mathrm{~mm})$; feuilles plus petites de $3-6(7) \mathrm{cm}$ de long et 1-2,5(3,5) cm de large. . . . . . . . 7. P. kirkii

2. Inflorescences $\sigma^{\prime}$ et $\bigcirc$ dissemblables, les $\sigma^{\prime \prime}$ spiciformes, robustes et denses, les o globuleuses. . . . . . . . . . . . . . 8. P. staudtii

8. Face inférieure des feuilles tomenteuse à poils fins courbés et entremêlés . . . . . . . . . . . . . . . . . . . . var. staudtii

8. Face inférieure des feuilles pubescente à poils apprimés raides .. . . . . . . . . . . . . . . . . . . . var appressa 